\title{
Improved constraints on non-Newtonian forces at 10 microns
}

\author{
Andrew A. Geraci ${ }^{1}$ * Sylvia J. Smullin ${ }^{1}$ David M. Weld ${ }^{1}$ 团 John Chiaverini ${ }^{1}$ 思 and Aharon Kapitulnik ${ }^{1,2}$ \\ ${ }^{1}$ Department of Physics, Stanford University, Stanford, CA 94305 and \\ ${ }^{2}$ Department of Applied Physics, Stanford University, Stanford, CA 94305
}

(Dated: October 22, 2018)

\begin{abstract}
Several recent theories suggest that light moduli or particles in "large" extra dimensions could mediate macroscopic forces exceeding gravitational strength at length scales below a millimeter. Such new forces can be parameterized as a Yukawa-type correction to the Newtonian potential of strength $\alpha$ relative to gravity and range $\lambda$. To extend the search for such new physics we have improved our apparatus utilizing cryogenic micro-cantilevers capable of measuring attonewton forces, which now includes a switchable magnetic force for calibration. Our most recent experimental constraints on Yukawa-type deviations from Newtonian gravity are more than three times as stringent as our previously published results, and represent the best bound in the range of $5-15 \mu \mathrm{m}$, with a $95 \%$ confidence exclusion of forces with $|\alpha|>14,000$ at $\lambda=10 \mu \mathrm{m}$.
\end{abstract}

PACS numbers: $04.80 . \mathrm{Cc}$

\section{INTRODUCTION}

A number of theories of physics beyond the Standard Model suggest that new physics related to gravity may appear at sub-millimeter length scales. For example, light moduli from string theory [1, 2] or exotic particles in "large" extra dimensions [3, 4, 5] can mediate forces exceeding gravitational strength that can be observed in a tabletop experiment. Such phenomena can be parameterized as a Yukawa-type correction to the Newtonian potential of strength relative to gravity $\alpha$ and range $\lambda$. For two masses $m_{1}$ and $m_{2}$ separated by distance $r$, the gravitational potential is modified to

$$
V(r)=-G_{N} \frac{m_{1} m_{2}}{r}\left(1+\alpha e^{-r / \lambda}\right) .
$$

To search for such new forces, we have improved our cryogenic apparatus utilizing silicon micro-cantilevers with attonewton force sensitivity [6, 7], which now includes a magnetic method for force calibration. The cantilever is loaded with a rectangular gold prism fabricated by focused-ion-beam milling that serves as a test mass for the experiment. The driving (source) mass is moved horizontally beneath the cantilever at a nominal vertical face-to-face separation of $25 \mu \mathrm{m}$. The force between the masses is deduced from the displacement of the cantilever as measured by a fiber-coupled laser interferometer. We perform the measurement at the cantilever resonance frequency, typically of order $300 \mathrm{~Hz}$, while the mechanical driving motion occurs at a sub-harmonic, typically onethird. This is achieved by implementing a density mod-

\footnotetext{
*aageraci@nist.gov Present address: National Institute of Standards and Technology, Boulder, CO 80305

${ }^{\dagger}$ Present address: Makani Power, Inc. Alameda, CA 94501

${ }^{\ddagger}$ Present address: Department of Physics, Massachusetts Institute of Technology , Cambridge, MA 02139

$\S$ Present address: Los Alamos National Laboratory, Los Alamos, NM 87545
}

ulation in the drive mass, consisting of alternating gold and silicon sections.

For the magnetic calibration, a $\mathrm{Co} / \mathrm{Pt}$ multi-layer film is deposited on the test mass. The permanent magnetic moment couples to a magnetic field gradient produced by current flowing across the meandering gold sections in the drive mass device. The current is turned off for the Yukawa-force search. A shield composed of high-magnetic-permeability material encloses the cryostat to prevent the Earth's field from magnetizing the drive mass. The amplitude and phase of a magnetic or Yukawa signal will change in a predictable way as we vary the equilibrium position of the drive mass oscillation. We utilize this scanning technique as an additional handle to distinguish a signal from background forces. In this paper, the newest data and error analysis are described, and the latest experimental constraints on Yukawa-type deviations from Newtonian gravity are presented. Finally, future directions are discussed.

\section{EXPERIMENTAL SETUP}

Many of the details of the experimental probe and vacuum cryostat are described in previous work [6, 7, 8$]$. The single-crystal silicon cantilevers are $250 \mu \mathrm{m}$ long, 50 $\mu \mathrm{m}$ wide, $0.3 \mu \mathrm{m}$ thick, and have a spring constant $k$ of approximately $0.0062 \mathrm{~N} / \mathrm{m}$. The thermal-noise-limited force sensitivity at $10 \mathrm{~K}$ is approximately $200 \mathrm{aN} / \mathrm{Hz}^{1 / 2}$ for a typical low-temperature quality factor $Q$ of 80,000 . The displacement of the cantilever beam is measured by using a fiber-coupled laser interferometer where a FabryPerot cavity is created between the end of the fiber optic and the cantilever loaded with a test mass [9]. The force $F$ on the cantilever is deduced from the measured displacement $z$, which is enhanced on-resonance by the large quality factor: $z=F Q / k$. The $1.5 \mu \mathrm{g}$ test masses measure $54 \times 54 \times 27 \mu \mathrm{m}^{3}$ and are cut from the edge of a $27 \mu \mathrm{m}$ thick gold foil using a $20 \mathrm{nA}$ gallium focusedion-beam (FIB). This technique produced masses with 
more regular shapes than those used in previous versions of the experiment 7] . A SEM image of a FIB-cut mass attached to a cantilever is shown in the inset of Fig. 1] The improvement allows a flatter surface to be presented to the drive mass, allowing more force sensitivity, and results in an improved interferometer signal. The driving (source) mass is mounted on a piezoelectric bimorph which actuates in the $y$-direction (as shown in Fig. 1) beneath the cantilever with an amplitude of $\sim 120 \mu \mathrm{m}$ about its equilibrium position. The drive mass consists of alternating $100 \mu \mathrm{m}$-wide bars of gold and silicon that are approximately $100 \mu \mathrm{m}$ deep and $1 \mathrm{~mm}$ long. As the bimorph oscillates with a sinusoidal motion, a time-varying gravitational force is exerted on the cantilever which can occur at the driving frequency of the bimorph as well as at higher harmonics, depending on the drive amplitude and spatial orientation of the test and drive masses. Simulation indicates a maximal gravitational coupling at the third harmonic of the drive frequency for a bimorph amplitude of $133 \mu \mathrm{m}$, with only a slight reduction $(\sim 10 \%)$ at $120 \mu \mathrm{m}$ [10]. A gold-coated silicon nitride shield membrane separates the cantilever from the drive-mass and provides attenuation of electrostatic and Casimir background forces from the oscillating drive mass. The drive mass is covered with a smooth $1 \mu \mathrm{m}$-thick plane of silicon, followed by aluminum oxide and gold to further suppress modulations in electrostatic or Casimir forces associated with the sections of alternating density. A schematic (not to scale) showing the cantilever, test and drive masses, shield, and piezoelectric bimorph actuator is shown in Fig. 1. A piezoelectric stack actuator is also included near the base of the cantilever to allow tests with deliberate excitation and to facilitate interferometer offset control and characterization. The voltage from the signal photodiode in the interferometer and the voltage applied to the bimorph actuator are recorded at 10 $\mathrm{kHz}$ on a data acquisition (DAQ) device connected to a PC. The cantilever signal (at the third harmonic of the drive frequency) is averaged with respect to the phase of the drive signal. A series of time records is collected for each data point. An FFT of the interferometer data is performed to determine the amplitude and phase at the third harmonic of the drive signal.

The amplitude and phase of either a magnetic or Yukawa signal will change in a predictable way as we vary the $y$-equilibrium position of the drive mass oscillation in situ. The $x, y$, and $z$-separation between the drive mass and test mass is controlled by a partially $(y, z)$ motorized manipulator stage at the top of the cryostat, and the tilt and $x, y$, and $z$-separation between the masses are detected by capacitive sensors [6, 7]. The expected Yukawa signal for a typical vertical mass separation and bimorph amplitude is shown in Fig. 2, The vertical axis shows the calculated amplitude and phase of a Yukawa ( $\alpha=1000, \lambda=18 \mu \mathrm{m})$ force at the third harmonic of the drive (which equals the resonance frequency of the cantilever). The horizontal axis shows the $y$-equilibrium position of the oscillation. Note that the third harmonic

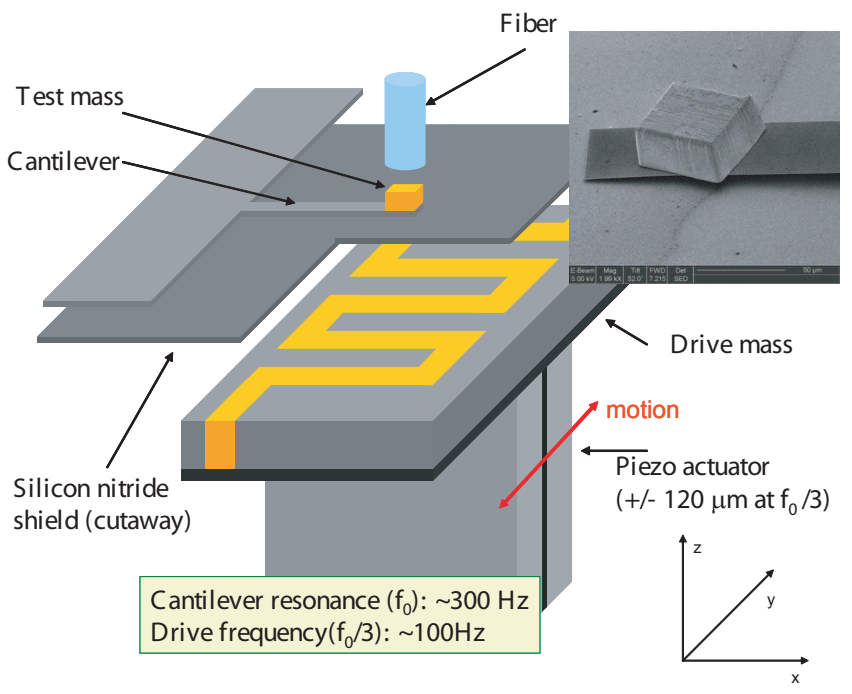

FIG. 1: (color online). A schematic (not to scale) showing the cantilever, test and drive masses, shield, and piezoelectric bimorph actuator. Figure is adapted from Ref. [11]. Not shown are the metallization of the shield and drive mass ground plane. A Fabry-Perot cavity is formed between the bottom of the optical fiber and top of the test mass and is used to interferometrically measure the cantilever displacement. The coordinate axes shown in the lower inset are used throughout the paper to describe the orientation of the drive mass and test mass. Upper inset: SEM micrograph of FIBfabricated test mass cut from $27 \mu \mathrm{m}$ thick gold foil attached to cantilever.

of the force (along with other odd harmonics) vanishes when the equilibrium position of the drive mass is such that a gold bar or silicon bar is centered underneath the cantilever. Conversely, the force is maximized when the interface of the bars is centered beneath the cantilever. This results in a $100-\mu \mathrm{m}$ spatial periodicity for the amplitude of a Yukawa-type signal.

\section{MAGNETIC CALIBRATION}

Having a magnetic force for calibration that is switchable in situ allows a great improvement in systematics by providing additional information about the expected phase structure of a Yukawa-type signal and by allowing multiple days of data to be analyzed together, as explained in Sec. [V] When current is applied in the meandering pattern of gold bars in the drive mass, the resulting magnetic field gradient above the pattern couples to a magnetic moment on the cantilever to provide a measurable force. This allows the relative position of the test mass with respect to the drive mass to be determined, as explained in the following subsection. When the current is turned off, for the Yukawa-measurement to have a clean background, it is necessary to eliminate or suppress the force due to the differing magnetic sus- 


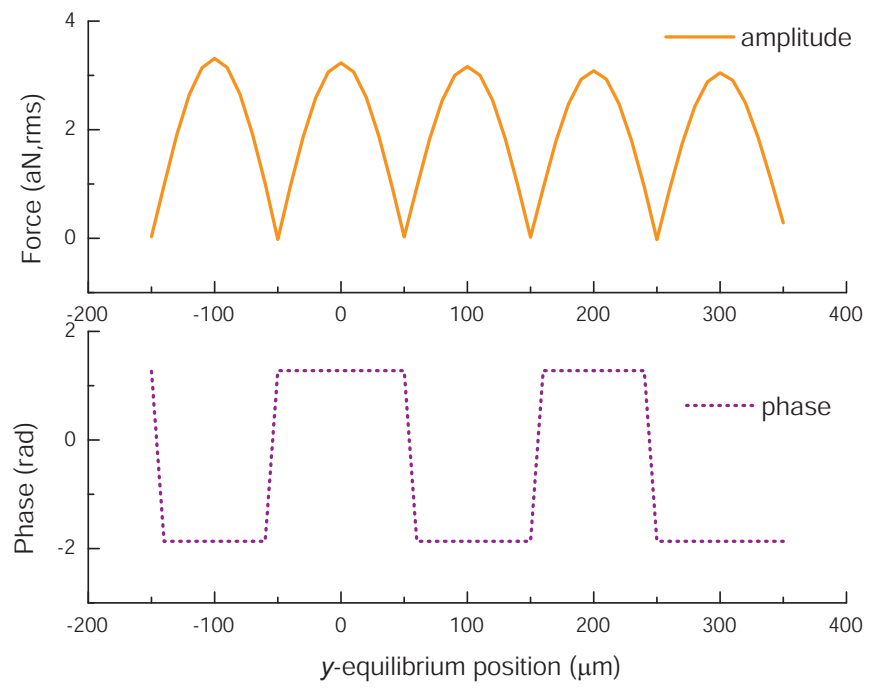

FIG. 2: (color online). Calculated vertical component of the Yukawa $(\alpha=1000, \lambda=18 \mu \mathrm{m})$ force amplitude and phase at the third harmonic of the drive frequency $(3 \omega)$, for $119 \mu \mathrm{m}$ of bimorph amplitude and $31.5 \mu \mathrm{m}$ edge-to-edge $z$-separation between masses with $6 \mu \mathrm{m}$ tilt across the drive mass in the scanning $(y)$ direction. The horizontal axis shows the $y$ equilibrium position of the drive mass oscillation. For reference, the $100 \mu \mathrm{m}$-wide gold bars are centered in the calculation at $y=-250 \mu \mathrm{m}, y=-50 \mu \mathrm{m}, y=150 \mu \mathrm{m}$, and $y=350 \mu \mathrm{m}$, with silicon bars in between.

ceptibility of the gold and silicon bars in the drive mass when the drive mass becomes magnetized in an ambient magnetic field such as the Earth's (see Sec. IIIB).

\section{A. Principle of the calibration}

The vertical force on the cantilever with permanent magnetic moment $\vec{m}_{c}$ in a magnetic field $\vec{B}$ is given by

$$
F_{z}=\left(\vec{m}_{c} \cdot \vec{\nabla}\right) B_{z}
$$

Also a torque on the cantilever $\vec{\tau}=\vec{m}_{c} \times \vec{B}$ can result in an effective force

$$
F_{z}^{\tau}=\tau_{z} / l_{c}
$$

where $l_{c}$ is the length of the cantilever. Simulations are performed to model the magnetic field from current flowing in the drive mass pattern, and the magnitude of the magnetic moment can be deduced from Eqs. (213) and the measured force on the cantilever, provided the direction of the magnetic moment is known. If the direction of the net moment is uncertain, only an approximate magnitude can be determined (see Fig. 3).

As we scan the $y$-equilibrium position of the oscillation in situ, the magnetic signal at the third harmonic of the motion frequency varies with a periodicity of $200 \mu \mathrm{m}$, corresponding to the spacing between the gold bars. In

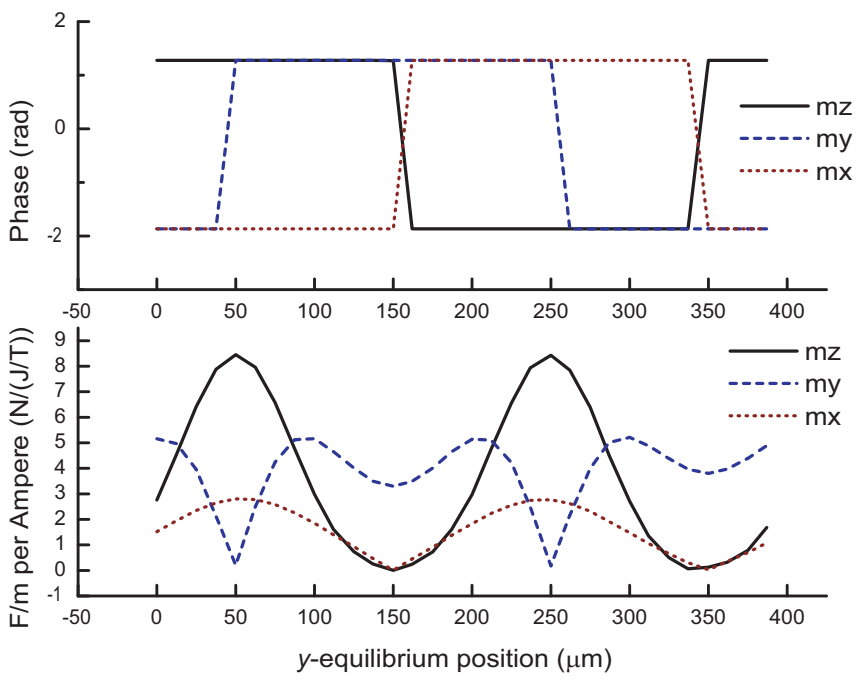

FIG. 3: (color online). Calculated magnetic force at the third harmonic of the drive frequency, per unit magnetic moment per ampere of current through the meander for varying $y$ equilibrium position of the drive mass oscillation. Results are shown for $119 \mu \mathrm{m}$ of bimorph amplitude and $30 \mu \mathrm{m}$ face-toface $z$-separation, for net magnetic moment in the $z, y$, and $x$ directions, respectively.

particular, as the $y$-equilibrium position of the drive mass oscillation is scanned, the amplitude of the force on the cantilever goes through a series of maxima and minima with a separation of $200 \mu \mathrm{m}$ between adjacent maxima and minima. Correspondingly, the phase of the force on the cantilever experiences a shift of $\pi$ across each magnetic minimum. The particular locations of the maxima and minima with respect to the position of the gold bars depends on the direction of the net magnetic moment. This is in contrast to the Yukawa signal, for which the maxima occur when the equilibrium position of the drive mass oscillation is such that the test mass is centered over the interface between the gold and silicon bars. The calculated magnetic force on the cantilever per ampere of current in the drive mass per unit magnetic moment is shown in Fig. 3 for the cases of a net moment aligned in the $x, y$, or $z$ directions, respectively. The vertical separation between the drive and test masses is taken to be 40 $\mu \mathrm{m}$ and the bimorph amplitude is $119 \mu \mathrm{m}$. The horizontal axis shows the $y$-equilibrium position of the bimorph oscillation.

To obtain a proof-of-principle measurement of the magnetic response, an early test was performed with a magnetic test mass consisting of a gold rectangular prism coated with $100 \mathrm{~nm}$ of nickel [7]. The $y$-equilibrium position of the drive mass oscillation was varied across the entire drive mass pattern as the force on the cantilever was studied with and without current running through the drive mass meander. With current in the drive mass meander, the force occurred with a $200 \mu \mathrm{m}$ periodicity as expected, corresponding to the spacing between the gold 
bars carrying current. With no current flowing across the drive mass, the force occurred with a $100 \mu \mathrm{m}$ periodicity and is due to the magnetization of the drive mass in the ambient earth's magnetic field. It is necessary to eliminate or suppress this susceptibility-dependent force in order to detect a new non-Newtonian signal.

The phase of the magnetic force both with and without current flowing across the drive mass is identical modulo $\pi$, as seen in earlier work [7] and verified in simulation. Simulation indicates that the phase of any Yukawa signal will also be identical to the phase of the magnetic force modulo $\pi$. This is a useful handle in the identification of a Yukawa force (see Sec. V).

\section{B. Magnetic shield}

In order to minimize the effect of the Earth's field, we employ a dual-layer magnetic shield assembly consisting of 0.050 " thick high magnetic permeability material provided by Amuneal Corporation. The shield is designed so it can be placed in position surrounding the cryostat. For data collection the cryostat is hung from the ceiling and hangs freely within the inner diameter of the magnetic shield. Numerical simulations performed by Amuneal Corporation [12] indicated an expected transverse shielding factor of 260 and longitudinal shielding factor of 160 . At Stanford, the earth's magnetic field is primarily vertical with a $z$-component of $45 \mu \mathrm{T}$, so the longitudinal figure is relevant. The shield is degaussed prior to the experimental runs.

\section{Co/Pt multilayer films}

The ideal magnetic moment would have a known magnitude and direction. Cobalt/platinum multi-layer films have been shown to have out-of-plane anisotropy for limited ranges of cobalt and platinum thickness [13, 14]. In order to obtain out-of-plane anisotropy the cobalt film thickness must be between $2.5 \AA$ and $11.5 \AA$. The platinum spacing layers must also be larger than $\sim 8 \AA[13]$. For the test masses, $3 \AA \mathrm{Co} / 15 \AA \mathrm{Pt} / 3 \AA \mathrm{Co} / 30 \AA$ $\mathrm{Pt}$ is sputtered on a $3 \mathrm{~mm}$ square gold foil substrate, on top of a seed layer of $100 \AA \mathrm{Pt}$ deposited at $350 \mathrm{C}$. Increasing the platinum thickness from 11 to $15 \AA$ resulted in improved out of plane anisotropy. The foil is then inserted into an MPMS-SQUID [15], and a hysteresis loop is studied for the film magnetized both out-of-plane and in-plane for fields of $-0.318 \mathrm{MA} / \mathrm{m}$ to $0.318 \mathrm{MA} / \mathrm{m}$. The coercive field is around $0.056 \mathrm{MA} / \mathrm{m}$ and full saturation occurs above $0.24 \mathrm{MA} / \mathrm{m}$. The results shown in Fig. 4 indicate an out-of-plane easy axis, although the in-plane magnetization loop also shows significant hysteresis. We attribute this to the imperfections on the foil surface as well as its curvature and roughness. Due to the nonideality, the data analysis of the experiment assumes that the direction of the magnetic moment is an unknown pa-

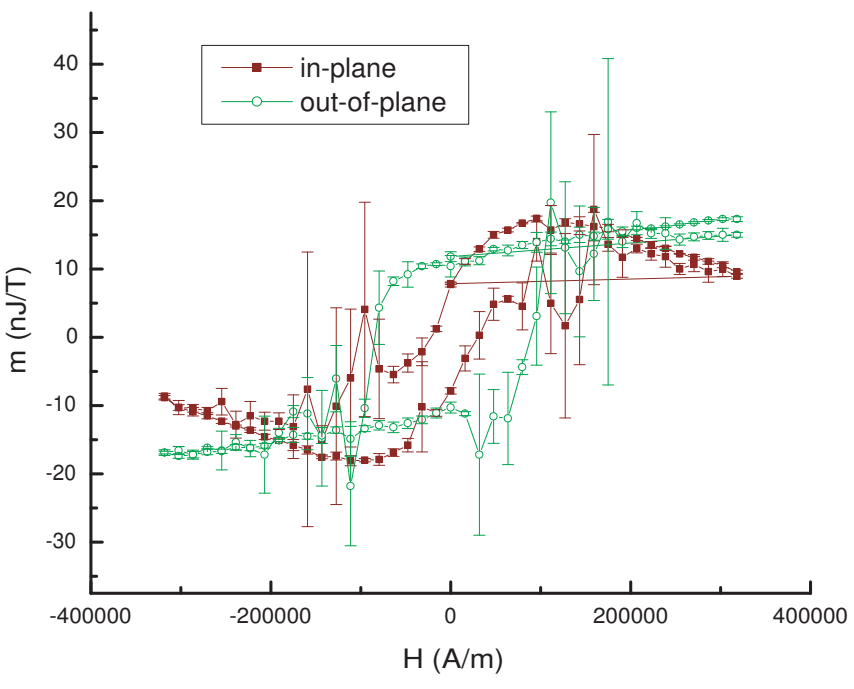

FIG. 4: (color online). Magnetic moment measured by MPMS-SQUID for deposition of $3 \AA \mathrm{Co} / 15 \AA \mathrm{Pt} / 3 \AA \mathrm{Co} /$ $30 \AA \mathrm{Pt}$ on gold foil substrate, including seed layer of $100 \AA$ $\mathrm{Pt}$ deposited at $350 \mathrm{C}$, for in-plane and out-of-plane magnetization.

rameter. However the magnitude of the moment can be reasonably well controlled as discussed in the following subsection.

\section{Magnetic Mass preparation}

The force due to the differing magnetic susceptibility of gold and silicon depends on the ambient field $B_{0}$ which magnetizes the drive mass. $B_{0}$ gets a contribution from the Earth's field, but also may be enhanced by the local magnetic environment. In particular the magnetic field emanating from the ferromagnetic layer on the test mass contributes to the drive mass magnetization. The differential volume-magnetic-susceptibility of gold and silicon $\Delta \chi$ is $3 \times 10^{-5}$, leading to a differential magnetization of order $\Delta M=\frac{1}{\mu_{0}} \Delta \chi B_{0}$. The correction to the local magnetic field and its gradient near the drive mass surface can be estimated by integrating the induced magnetic dipole moment per unit volume over the volume of the drive mass. Very roughly, the differential magnetic force experienced when the magnetic test mass is above gold versus above silicon can be approximated as

$$
\Delta F \approx m_{c} V_{\mathrm{eff}} \mu_{0} \Delta M / r^{4}
$$

where $r$ is the distance to the test mass magnetic film from the center of the drive mass bar, $m_{c}$ is the magnetic moment on the cantilever, and $V_{\text {eff }}$ is the effective volume of the drive mass within range to contribute to the signal. To obtain a precise estimate of this force the net direction of the magnetic moment on the cantilever must be known; otherwise only order-of-magnitude estimates are possible. The magnetic measurements described in Refs. [7, 10] 
can be explained by Eq. (4) to within uncertainties, and therefore Eq. (41) can be used to determine the target amount of magnetic material on the test mass.

To obtain a large enough force signal for a reasonable drive mass current $(10 \mathrm{~mA})$, while also having negligible magnetic susceptibility force with the current off, it is sufficient to have a magnetic shield attenuation factor greater than 100 and a total moment $m$ satisfying $5 \times 10^{-15} \mathrm{~J} / \mathrm{T}<m<5 \times 10^{-13} \mathrm{~J} / \mathrm{T}$. For two layers of $3 \AA \mathrm{Co} / 15 \AA \mathrm{Pt}$, measurements suggest an area of $(25 \mu \mathrm{m})^{2}$ yields a moment of $5 \times 10^{-13} \mathrm{~J} / \mathrm{T}$. The foil is inserted into the FIB and sections of the magnetic layer near an edge of the foil are removed with a $100 \mathrm{pA}$ ion beam. $12 \mu \mathrm{m}$ squares of magnetic material are left in the locations that will become the centers of the test masses and should result in a moment of approximately $1 \times 10^{-13} \mathrm{~J} / \mathrm{T}$. A high-current $20 \mathrm{nA}$ ion beam is then used to cut away the edge of the foil to produce a clean edge. The test masses are defined using the high current beam. Care is taken to ensure that the high current beam does not directly etch any magnetic material, as it has a tendency to deposit material as the beam cuts. A thin film of gold covers the magnetic patches on the test mass due to this effect. The masses are completely free except for a small "tail". At this point the masses are reinserted into the SQUID and re-magnetized outof-plane. This re-magnetization is performed to eliminate the effects of any demagnetization that may have occurred from heating while the high current beam cuts through the foil. The resolution of the SQUID is insufficient to measure the magnetic moment of a single test mass. However, the bulk foil can be seen to attain the same value of magnetic moment as originally measured. After re-magnetization the masses are mechanically extracted from the foil using non-magnetic tungsten probe tips. In this way subsequent heating from the ion beam can be avoided. The masses are epoxied onto the cantilever using Hysol-Loctite 1C epoxy [16] and again only handled with a tungsten probe tip to avoid magnetic contamination.

\section{NOISE AND BACKGROUND SOURCES}

Thermal Noise. The minimum detectable force due to thermal noise at temperature $T$ is

$$
F_{\text {min }}=\sqrt{\frac{4 k_{B} T B k}{\omega_{0} Q}},
$$

where $B$ is the bandwidth of the measurement, $k$ is the cantilever spring constant, and $f_{0}=\omega_{0} /(2 \pi)$ is its resonance frequency. This represents a fundamental limit on the sensitivity of the measurement technique.

Vibrational Noise. At the cantilever resonance frequency, assuming a $4 \%$ non-linearity in the bimorph and $Q$ of $10^{5}$, we estimate the required internal vibration isolation (between the bimorph and cantilever) to be
$1.3 \times 10^{5}$, employing a simple analytic model [10]. Two sets of spring-mass stages isolate the platform on which the bimorph is mounted from the mount holding the cantilever, providing a factor of $10^{6}$ attenuation at $100 \mathrm{~Hz}$ and $10^{8}$ attenuation at $300 \mathrm{~Hz}$ [7]. The mass stages are also connected by thin wires in addition to the springs to operate the various sensors and piezo-electric stack. If these wires become too taut during the cooling of the probe, the vibration isolation system can become compromised. A direct measurement of the vibrational coupling at $300 \mathrm{~Hz}$ is unfortunately not possible due to the operating frequency range of the bimorph. The cryostat is hung from $1 \mathrm{~Hz}$ springs from a thick concrete ceiling to attenuate unwanted external vibration at $300 \mathrm{~Hz}$.

Electrostatics. The stainless steel frame which holds the cantilever wafer is grounded to the main probe ground, so that together with the metallized shield, a Faraday cage surrounds the cantilever. Any voltage difference $\Delta V$ built up between the cantilever and shield will result in attractive force between them of magnitude $F=\epsilon_{0} A(\Delta V)^{2} / 2 d^{2}$ where $A$ is the area of the cantilever surface, and $d$ is the separation between the cantilever and shield. Any built up charge on the cantilever can produce an electrostatic force. In a measurement using a similar shield, the shield motion at $300 \mathrm{~Hz}$ was found to have an upper bound of approximately $\delta d \sim 1 \mathrm{pm}[8,11]$. Therefore voltage differences of $100 \mathrm{mV}$ can drive the cantilever above thermal noise. Alternatively, if a spurious electrical voltage signal $V_{0} \sin \omega_{0} t$ with $V_{0}>100 \mu \mathrm{V}$ leaks into the shield, the cantilever can be driven above thermal noise. In either case the signal could not be mistaken for a new Yukawa force since it would not vary with the spatial periodicity of the drive mass pattern.

Casimir force. There is a relatively large $\left(\sim 3 \times 10^{-16}\right.$ N) Casimir force present at all times between the cantilever and shield membrane. If constant, it does not impede a force measurement at the cantilever resonance frequency. There is also a Casimir interaction between the shield membrane and drive mass ground plane. The oscillating bimorph can drive the shield membrane into motion, and due to piezo non-linearity, can excite it at the cantilever resonance frequency. The shield membrane is sufficiently stiff so that the modulation of the Casimir force on resonance does not excite the cantilever beyond the level of thermal noise $\sim 10^{-18} \mathrm{~N}$ for our measurement bandwidth [6, 7]. Even if the shield were driven enough to excite the cantilever on resonance above thermal noise, the signal would not exhibit the $y$-equilibrium position dependence that a Yukawa signal would have, and therefore could not be erroneously interpreted as a new force.

One can also ask whether the shield membrane and drive mass ground plane are sufficient to screen the direct Casimir force between the drive mass and test mass, given the finite conductivity, plasma frequency, and thickness of the gold coating. In fact, the drive mass ground plane alone is enough to prevent the differential Casimir force from the gold and silicon drive mass sec- 
tions from being transmitted at a detectable level. Following Ref. [17] we employ a reflection-based model for computing the Casimir force between two metallic walls. We obtain a reduction factor $\eta_{F}$, (defined precisely in Ref. [17]) which describes the reduction of the Casimir force at small separations and for finite conductivity. The parameter $\eta_{F}$ is defined as $F_{C}=\eta_{F} F_{P}$ where $F_{P}$ is the perfect conductor result. We take a gold test mass of thickness $30 \mu \mathrm{m}$ as one mirror, and consider the differential Casimir force for the situations of the other mirror being $25 \mu \mathrm{m}$ away and composed either of $0.1 \mu \mathrm{m}$ thickness $\mathrm{Au}$ (corresponding to the drive mass ground plane) or $100.1 \mu \mathrm{m}$ thickness $\mathrm{Au}$ (corresponding to the drive mass ground plane plus gold bar). We find that the difference in $\eta_{F}$ is 0.0028 , corresponding to a differential Casimir force of $2.4 \times 10^{-20} \mathrm{~N}$, allowing $\alpha=1$ to be probed at $\lambda=20 \mu \mathrm{m}$, which is well below the sensitivity of the experiment. The shield membrane attenuates this Casimir interaction even further, rendering the effect negligible.

Magnetic background. Apart from those discussed in Sec. III, there are other mechanisms for a magnetic coupling that occur with the drive mass spatial periodicity. These include the susceptibility-induced interaction between the drive mass and the gold part of the test mass and eddy currents produced in the drive mass as it oscillates in the remnant ambient field. Also, any charge built up in the silicon sections of the drive mass can generate a weak magnetic field as the drive mass moves. All of these mechanisms are too weak to produce a measurable signal in this experiment.

Interferometer noise. Sources of electronic and optical noise in the interferometer are discussed in Refs. [8, 11]. Electronic noise consists of shot noise from the laser, and to a lesser extent Johnson noise from the $10 \mathrm{M} \Omega$ feedback resistor and fluctuations in the laser current supply, and combines to produce typically a few $\mu \mathrm{V} / \mathrm{Hz}^{1 / 2}$. Optical noise can be produced by stray reflections in the interferometer as well as mechanical shaking of the fiber. Modulation of the laser at frequencies over $100 \mathrm{MHz}$ serves to reduce stray reflections by reducing the coherence length of the laser [18, 19]. Mechanical shaking of the fiber can mimic cantilever displacement, along with any electrical coupling at harmonics of the bimorph drive signal. The amplitude of these noise sources is assessed by measuring with the drive frequency off-resonance from a subharmonic. However, this voltage noise is not expected to vary periodically as the $y$-equilibrium position of the drive mass oscillation is scanned and thus does not mimic a Yukawa-like force.

\section{DATA}

The general cooling-down procedure and data acquisition technique are discussed at length in Refs. [7, 8]. The resonance frequency $f_{0}$ of the cantilever ranged from 324.082 to $324.136 \mathrm{~Hz}$ while near base temperature dur- ing the course of the experimental run which is the central subject of this paper. The base temperature of the probe ranged from approximately $11-13 \mathrm{~K}$. The quality factor as measured using the ring-down technique (see for example Ref. [8]) was approximately 85,000. The spring constant $k$ of $0.0062 \mathrm{~N} / \mathrm{m}$ was inferred from the measured resonance frequency and calculated mass of the test mass. The effective temperature of the cantilever is deduced from $k$ and the square of the amplitude spectrum near the cantilever thermal peak through the equipartition theorem. This effective noise temperature was typically measured to be $20-25 \mathrm{~K}$ at low laser power, about $10 \mathrm{~K}$ above the base probe temperature, as was typically seen in previous work [7]. The quality factor and effective temperature of the cantilever were both found to depend on the incident laser power. The laser power was reduced below $1 \mu \mathrm{W}$ before temperature and quality factor stopped changing. The low-temperature fringe height (as defined in TableI) was correspondingly reduced to approximately $250 \mathrm{mV}$. The voltage noise on the interferometer, as assessed by tuning the piezo driving frequency $100 \mathrm{mHz}$ off resonance, was at the level of $10^{-18} \mathrm{~N}$ for the working fringe height and quality factor. For the gravity force measurement, the quality factor of the cantilever was adjusted with feedback 7] to $9500-10000$, and the effective temperature with feedback was between $2-3$ $\mathrm{K}$. The thermal noise limited force sensitivity was $\sim 200$ $\mathrm{aN} / \sqrt{\mathrm{Hz}}$.

Prior to collecting data in search of non-Newtonian gravity (referred to hereafter as 'gravity data'), the approximate $x$ - and $y$-position of the drive mass with respect to the test mass is coarsely determined by using the magnetic force signal, and it is verified that the test mass is positioned over the central meandering region of the drive mass. Gravity data are then collected as a function of the $y$-equilibrium position of the drive mass oscillation for a given $z$-separation. For each point of gravity data collected, a magnetic force measurement is done at the same location by applying current across the drive mass. Then the magnetic force is scanned in the $y$-direction to determine the $y$-position of the closest magnetic minimum. In this way the relative $y$-position between gravity data points can be determined even in the presence of drifts in the $y$-capacitance sensor reading of a given $y$-position that can occur over the time scale of days, as demonstrated in earlier experimental runs with a nickel magnetic layer on the test mass [8]. Having the magnetic calibration (switchable in situ) thus permits multiple days of data to be analyzed together, a significant improvement over previous renditions of the experiment.

The data set is shown in Fig. 5 The magnitude and phase of the magnetic (drive mass current on) and possible Yukawa (drive mass current off) forces at the third harmonic are studied as the $y$-equilibrium position of the drive mass oscillation is varied. The 17 data records each consist of $24-120$ minutes of averaged data. The amount of averaging time chosen varies considerably due to the limited duty cycle in the experiment. Environ- 

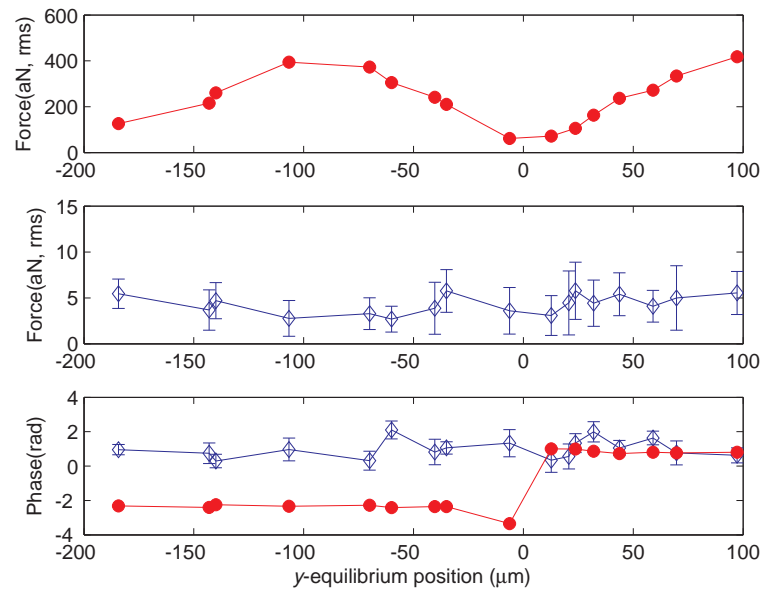

FIG. 5: (color online). Main data set showing statistical and systematic uncertainties in the measured force. Diamonds show signal with current off. Circles show signal with $10 \mathrm{~mA}$ current in the drive mass. Lines are guides to the eye. Error determination is described in Sec. VI] The $y$-coordinates shown for the data set on the horizontal axis are the displacements relative to one particular magnetic force minimum chosen as $y=0$. We note that these coordinates vary by an unknown offset with respect to those shown in the calculations in Figs. 2 and 3 .

mental noise and possibly the internal (bimorph-related) vibrational noise varied considerably over the course of the data run. By 'environmental noise' we here refer to noise that is not associated with the motion of the bimorph actuator. In particular, environmental noise may have come from the several nearby large scale construction projects that were underway during various stages of the data run. As a test of the environmental noise, measurements were taken with the bimorph-actuator turned off. Internal vibrational noise can in principle be caused by a short of the vibration isolation springs due to taut probe wiring. Although considerations are taken at room temperature when the system is open to prevent taut wiring, it can be difficult to ensure that no vibration isolation shorts develop after cooling. The amount of internal (bimorph-related) vibration noise can therefore vary based on the $x, y$, and $z$-separation and tilts of the bimorph stage with respect to the mass-stage holding the cantilever. The effects of vibration due to internal isolation shorts were examined with the test and drive masses far $(\sim 1 \mathrm{~mm})$ withdrawn from each other and the bimorph moving. These noise sources limited the amount of useful averaging time over the experimental run. The data in Fig. 5 were taken only at time periods when such noise was minimal. Since we are not searching for rareevent physics, it is legitimate to discard those other data for which large disturbances were clearly present on the system.

The phase of the magnetic force undergoes a change of $\pi$ at the magnetic force minimum at $y=0$. If a pure Yukawa signal were present, one expects to see two phase changes of $\pi$ in the drive mass current-off (abbreviated hereafter as 'current-off') data for every phase change in the magnetic (current-on) signal, and two force minima for every magnetic force minima. The $y$-extent of the data set should include three Yukawa phase changes, each separated by $100 \mu \mathrm{m}$ in the presence of such a force. The current-off data therefore shows no clear Yukawa signal in Fig. 5. In fact, the phase of the current-off data remains relatively constant over the data set. In the presence of thermal noise alone, the phase would be expected to be distributed randomly. The bound on any Yukawa signal associated with the data set is presented in Sec. VI. The strength of the magnetic moment can be roughly estimated from the current-on response. Simulation results indicate a magnetic moment of approximately $1.3-3.7 \times 10^{-14} \mathrm{~J} / \mathrm{T}$, depending on the direction of the moment.

The relatively constant phase of the current-off data could have a number of possible origins. The driving signal applied to the piezo actuator could have some small electronic leakage to the piezo near the cantilever. The internal vibrational noise due to the bimorph oscillation can tend to occur at a common phase given a common mechanism. Bimorph non-linearity is also responsible for some shield motion at the cantilever resonance frequency. Although the associated modulation of the Casimir force is too small to be observed, excess charge on the cantilever could couple to small displacements of the shield membrane driven by the bimorph. The patch effect can produce local potential variations in the gold coating on the shield membrane which can vary with respect to the potential on the gold test mass 20]. In any case, the expected $100 \mu \mathrm{m}$ spatial periodicity of a Yukawa-like signal can be distinguished from a background force with a relatively constant phase. Therefore, an accurate bound on a Yukawa-like interaction can be determined even in the presence of such a background force, as discussed in Sec. VI,

\section{ERROR ANALYSIS}

The experimental error in the determination of either a bound on or discovery of a Yukawa-type correction to Newtonian gravity can be sorted into two types. First there are the statistical and systematic uncertainties in the measurements of the force $F_{\text {meas }}$. The second type is the systematic error which enters the finite-element calculation of the expected Yukawa force signal $F_{Y}$. We treat the experimental uncertainties in a Monte-Carlo fashion, based on the method described in Ref. [7]. In order to obtain the function $\alpha(\lambda)$ for a Yukawa force that best fits the measured data we consider a least-squares fit of the real and imaginary parts of the data to a set of calculated forces in the Monte-Carlo simulation. The four fitting parameters, as a function of $\lambda$, are the re- 
TABLE I: Uncertainty in measured values of forces. The voltage on the interferometer is given by $V_{\text {intf }}(d)=V_{c}-$ $\frac{V_{\mathrm{pp}}}{2} \cos (4 \pi d / \lambda)$, where $d$ is the distance between test mass and the end of the optical fiber, and $\lambda$ is the laser wavelength. The fringe height $V_{\mathrm{pp}}=\left(V_{\max }-V_{\min }\right)$ and the fringe center $V_{\mathrm{c}}$ is the mean of $V_{\max }$ and $V_{\min }$. The fringe position is the deviation from the fringe center.

\begin{tabular}{lcccc}
\hline \hline & & & & \\
Parameter & Value & Error & Units & $d F \%$ \\
\hline & & & & \\
Fringe height & $.228-.472$ & $.002-.004$ & $\mathrm{~V}$ & 1 \\
Fringe position & 0 & 3 & $\%$ & 0.2 \\
$f_{0}$ & $324.082-324.136$ & .002 & $\mathrm{~Hz}$ & 0.7 \\
$Q$ & $9500-10100$ & 400 & - & 4.1 \\
$k$ & .0062 & .0003 & $\mathrm{~N} / \mathrm{m}$ & 4.5 \\
Fiber alignment & 0 & 22 & $\mu \mathrm{m}$ & 12 \\
& & & & \\
\hline & & & & 13 \\
Total
\end{tabular}

sult $\alpha$, an offset variable $y_{0}$ to account for the lack of absolute $y$-position information between the drive mass pattern and test mass due to the unknown direction of the magnetic moment, and the real and imaginary parts of a constant offset force $\left(R_{0}, I_{0}\right)$. It is reasonable to include a constant offset force to account for vibrational or other-type backgrounds that do not depend periodically on the $y$-equilibrium position of the drive mass.

\section{A. Uncertainty in the measured force}

Statistical errors. The statistical error on each data point comes primarily from thermal noise at the effective cantilever temperature and is the most significant contribution $(90 \%+)$ to the total $1 \sigma$ error bars shown in Fig. 5 . The variation in the standard error reflects the amount of averaging time for each data point, which varies from 24 to 120 minutes. The statistical error decreases with the square root of the numbers of samples. There is a natural time scale for the duration of statistically dependent samples given by the ring-down time of the cantilever $\tau=\pi f_{0} Q$. We divide the data records into subsections of length 30 seconds $\sim 3 \tau$ and study the cross correlation between these subsections to verify their statistical independence, by the method discussed in Ref. 7].

Systematic errors in the measured force. The systematic errors in the measured force are listed in Table [) The error is dominated by the uncertainty in the exact position of the fiber over the top surface of the test mass. Considering the cantilever mode shape we estimate the corresponding $1 \sigma$ error in the measured force as $12 \%$. The uncertainty including other systematics in the force
TABLE II: Uncertainty in tilt and $z$-separation. Total tilts of the drive mass relative to the shield plane are expressed in vertical distance over the $1.8 \mathrm{~mm}$ length or $1.3 \mathrm{~mm}$ width of the drive mass.

\begin{tabular}{lccc}
\hline \hline & & & \\
Parameter & Value $1 \sigma$ Error & Units \\
\hline & & & \\
$z$-separation & & & \\
Shield-to-drive mass & $11-15$ & 1 & $\mu \mathrm{m}$ \\
Edge-edge between masses & $27-31$ & 3 & $\mu \mathrm{m}$ \\
Mean edge-edge separation & 29 & 3 & $\mu \mathrm{m}$ \\
MC Input & 29 & 3 & $\mu \mathrm{m}$ \\
Relative to the shield plane & & & \\
Total tilt in $y$ - $z$ plane & 2 & 5 & $\mu \mathrm{m} / \mathrm{length}$ \\
Total tilt in $x$ - $z$ plane & 3 & 5 & $\mu \mathrm{m} / \mathrm{width}$ \\
& & & \\
\hline \hline
\end{tabular}

measurement added in quadrature is approximately $13 \%$.

\section{B. Uncertainty in the calculated force}

Uncertainty in vertical separation and tilt. The calculated Yukawa force strongly depends on the vertical separation between the drive and test masses due to its exponential form. The tilt of the drive mass with respect to the shield wafer will also effectively increase the $z$-separation, by an amount that depends on the $x$ and $y$ position of the test mass with respect to the drive mass edges. Table Iindicates the experimental uncertainty in the $z$-separation and tilts. The tilts and $z$-separation are determined using methods described in previous work [7]. From this table, inputs to the Monte-Carlo are derived. The Monte-Carlo input for $z$-separation is the mean edgeedge separation, adjusted to account for the tilt in the drive mass silicon plane [10]. Variations in $z$-separation between data points are very similar to variations within a particular data point $(1 \mu \mathrm{m})$ and typically are less than the overall uncertainty in $z$-separation $(3 \mu \mathrm{m})$. Also for the tilts, variation of tilts across data points are much less than the overall uncertainty in the tilt. Therefore for the Monte-Carlo we can treat the $z$-separation and tilts of the entire data set as their mean value and add a Gaussian random error with their standard deviation. This is a reasonable approximation since the standard deviation in the measured tilt or $z$-separation over the 17 point data set is significantly less than the uncertainty in the determination of the tilt or $z$-separation at any one data point. A more detailed accounting of the determination of tilt and separation uncertainty appears in Ref. [10].

Uncertainty in bimorph amplitude. The mean bimorph amplitude recorded for the data set was $119 \mu \mathrm{m}$ with a standard deviation of $1.3 \mu \mathrm{m}$. The $1 \sigma$ uncertainty due 
TABLE III: Errors due to masses

\begin{tabular}{lc}
\hline \hline & \\
Parameter & $d \alpha \%$ \\
\hline & \\
Volume of Test Mass & 4.5 \\
Voids in Drive Mass & 2.5 \\
Drive mass polished Au/Si boundary & 1
\end{tabular}

TABLE IV: Uncertainty in Masses and Geometry

\begin{tabular}{lccc}
\hline \hline & & & \\
Parameter & Value & Error & Units \\
\hline & & & \\
Test Mass length & 53 & 1 & $\mu \mathrm{m}$ \\
Test Mass width & 54 & 1 & $\mu \mathrm{m}$ \\
Test Mass height & 27 & 1 & $\mu \mathrm{m}$ \\
Volume of Test Mass & 77000 & 3500 & $\mu \mathrm{m}^{3}$ \\
Density of gold & 19300 & 0 & $\mathrm{~kg} / \mathrm{m}^{3}$ \\
Total mass of test mass & 1.50 & 0.07 & $\mu \mathrm{g}$ \\
& & & \\
\hline
\end{tabular}

to the motion calibration is $10 \mu \mathrm{m}$. The method for calibrating the bimorph and for determining the error in bimorph amplitude is discussed at length in Ref. [8].

Uncertainty in geometry and density of masses. The uncertainty in the geometry of the drive mass is discussed at length in Ref. [8]. The uncertainty in the test mass geometry is determined by a combination of optical and SEM imagery. Table III lists their relative contribution to the uncertainty in $\alpha$, which is included as a multiplicative scaling error to the best fit results, using the method discussed in Ref. [7]. The effective length, width, and height shown in Table IV are determined by considering the approximately $84^{\circ}$ sloping profile of the test mass walls measured by the SEM and accounting for the extra mass present near the final-release corner. A SEM micrograph showing the mass attached to the cantilever after being used in the data set is shown in the inset of Fig. 1.

Uncertainty in relative y-position of data points. For the Monte-Carlo analysis to be optimally constraining, the relative $y$-separation of the data points must be known to within a few microns. The $y$-coordinate of each data point has a $1 \sigma$ uncertainty of $2.5 \mu \mathrm{m}$. This accounts for the uncertainty in the $y$-position of the magnetic minimum, as well as the uncertainty in the $y$-position at the data collection point.
Uncertainty in the expected phase of a Yukawa-type signal. Simulation indicates that the phase of any Yukawa signal will be identical to the phase of the magnetic force modulo $\pi$. The uncertainty in the expected phase (modulo $\pi$ ) of a Yukawa signal is determined from the uncertainty $(0.1 \mathrm{rad})$ in the measured phase of the magnetic calibration signal.

\section{Summary of inputs to the Monte-Carlo}

The set of geometrical inputs $(z$-separation and tilts in the $y-z$ and $x-z$ planes, respectively) for the Monte-Carlo calculation are summarized in Table II. For each of the values of $\lambda$ considered $(4,6,10,18,34,66 \mu \mathrm{m})$, the Yukawa force versus $y$-displacement is calculated for 320 elements of a Gaussian distribution of these geometrical parameters, with the means and standard deviations as indicated. For the mean value of bimorph amplitude, the corresponding $3 \omega$ force versus $y$-displacement is determined. For each of these curves, a least-squares fit to the measured forces and $y$-positions is performed, yielding the best-fit $\alpha, y_{0}, R_{0}$, and $I_{0}$. The measured force, measured $y$-position of the data points, and expected phase of the Yukawa type signal are then 'dithered' (with a Gaussian random value added) 200 times according to the Gaussian distribution of their statistical and systematic errors as described in the previous subsections. For the uncertainty in phase, an element from a Gaussian distribution is randomly assigned to the expected phase (modulo $\pi$ ) of a Yukawa type signal, according to the uncertainty in the phase of the measured magnetic signal. Each dithered set of data is then fit to yield a new best-fit value of $\alpha$. This results in a set of $320 \times 201=64,320$ least-squares fits of the data to the calculated curves, producing 64,320 best-fit values of $\alpha$ for each $\lambda$. Finally, these values of $\alpha$ are appropriately scaled to account for the uncertainty in the bimorph amplitude and the uncertainty in the volume of the drive and test masses, by using 50 samples taken from Gaussian distributions representing these uncertainties. The treatment of these multiplicative errors is further discussed in Ref. [10]. The end result is a histogram of $3,216,000$ best fit $\alpha$ for each of the six values of $\lambda$ explicitly considered. The numbers of samples are chosen to be sufficiently large so that the means and 95th percentiles of the histograms do not vary significantly with the numbers of samples.

\section{RESULTS}

The histogram resulting from the Monte-Carlo analysis for $\lambda=10 \mu \mathrm{m}$ is shown as an example in Fig. 6. To illustrate a typical fit of the force calculation to the data we consider an element near the mean of the histogram for $\lambda$ of $10 \mu \mathrm{m}$, corresponding to $\alpha=4450$. Figure 7 shows the real and imaginary parts of the data along with statistical and systematic error bars. Also shown is the best 


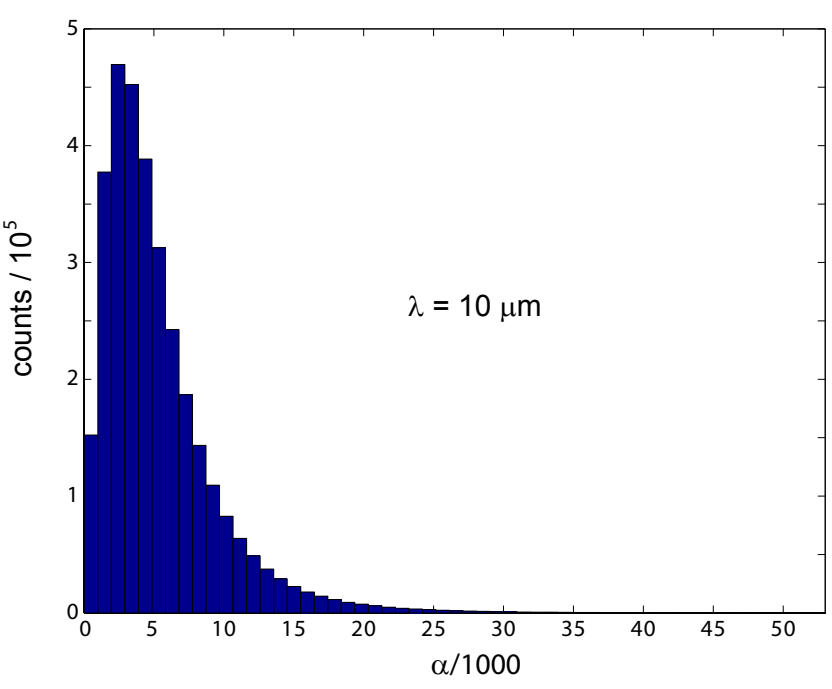

FIG. 6: (color online). Histogram of best-fit $\alpha$ results for $\lambda=10 \mu \mathrm{m}$.

fit Monte-Carlo result for comparison. Figure 8 shows the amplitude and phase of the data along with statistical and systematic error bars. The solid line shows the best fit Monte-Carlo result to the data. We note that the actual fit is performed on the real and imaginary parts of the data - the amplitude and phase are plotted for illustration. For reference, the dotted line indicates the best fit result after removing the constant offset force. The fit is performed with the offset included and the offset is subtracted afterwards. The data set is clearly best fit with a constant offset force included, as evidenced by the concentration of phase near $1 \mathrm{rad}$ for the entire data set. The fact that the best-fit phase does not vary much reflects the adding of the best-fit Yukawa component (with an amplitude of $0.5 \mathrm{aN}$ ) to the larger background best-fit offset rms force vector $(2.1 \mathrm{aN}, 3.1 \mathrm{aN})$.

The mean values of $\alpha$ and 95\% confidence exclusion bounds for Yukawa type forces are derived from the set of six histograms and listed in Table $\mathrm{V}$. The ratio of the $95 \%$ confidence limit to the mean value increases as $\lambda$ decreases, reflecting the increased significance of the uncertainties in $z$-separation and tilt. The means of each histogram represent the most likely value of any Yukawa force that may be present. Upcoming experiments will exhaustively re-examine this parameter space. The results represent an improvement of the constraints on Yukawa forces by approximately half an order-ofmagnitude over our previously published work [7]. We also note that the mean values of $\alpha$ from the histograms in Ref. [7] are now excluded at better than the $95 \%$ level by the present work. Our final results are shown in Fig. 9. We show only the $95 \%$ confidence exclusion results from Table $\mathrm{V}$

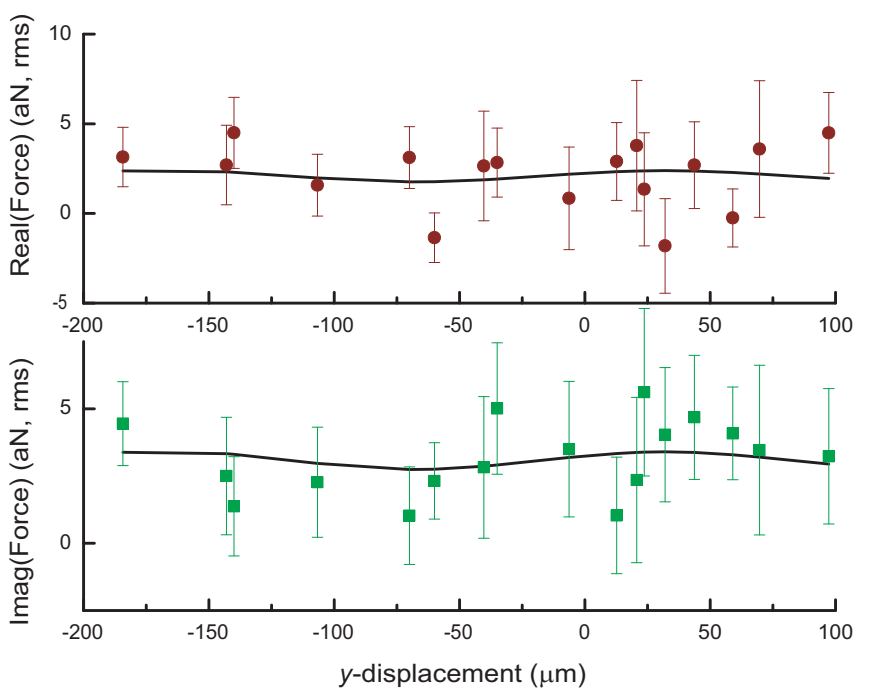

FIG. 7: (color online). Real and imaginary parts of measured force. A typical Monte-Carlo fit result (best fit Yukawa signal plus offset) for $\lambda=10 \mu \mathrm{m}, \alpha=4450$, is shown as a solid line. Error bars $(1 \sigma)$ on the measured forces are also shown.

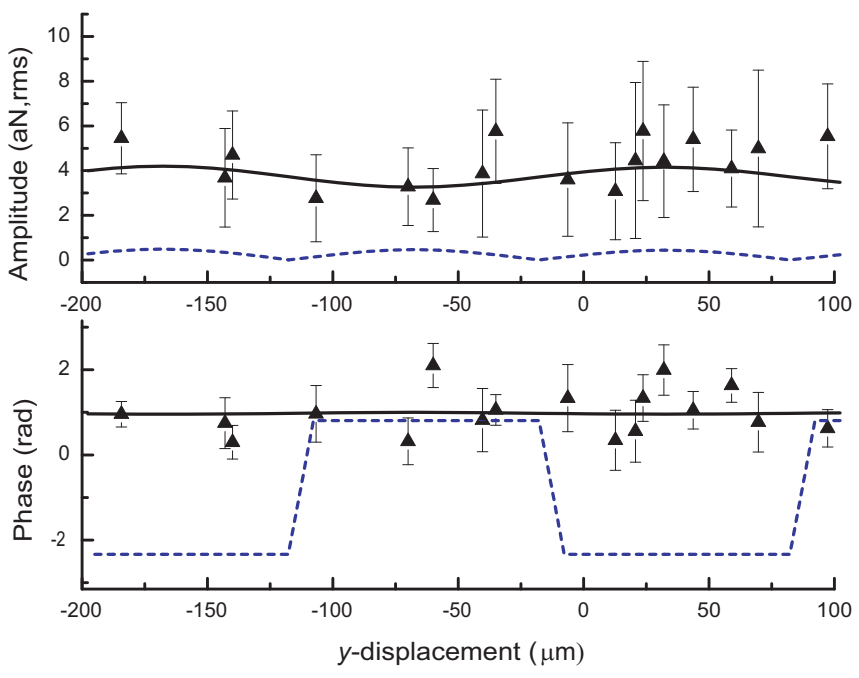

FIG. 8: (color online). Amplitude and phase of measured force. A typical Monte-Carlo fit result (best fit Yukawa signal plus offset) for $\lambda=10 \mu \mathrm{m}, \alpha=4450$, is shown as a solid line. Error bars $(1 \sigma)$ on the measured forces are also shown. Dotted line shows fit result with best-fit offset-force subtracted. The amplitude of the best-fit offset-force is significantly larger than the amplitude of the Yukawa component.

\section{FUTURE DIRECTIONS}

One limiting factor of the experimental run had to do with the duty cycle of the experiment. Improvements to the duty cycle could be made by adding more automation to the experiment or transferring the apparatus to a generally less noisy environment. A substantial improvement could involve the redesign of the cantilever 
TABLE V: Experimental limits on Yukawa forces

\begin{tabular}{ccc}
\hline \hline & & \\
$\lambda(\mu \mathrm{m})$ & Mean $(\mathrm{MC}) \alpha$ & $95 \%$ exclusion $\alpha$ \\
\hline & & \\
4 & $8.6 \times 10^{6}$ & $3.1 \times 10^{7}$ \\
6 & $1.6 \times 10^{5}$ & $4.6 \times 10^{5}$ \\
10 & $5.6 \times 10^{3}$ & $1.4 \times 10^{4}$ \\
18 & $5.1 \times 10^{2}$ & $1.1 \times 10^{3}$ \\
34 & $1.2 \times 10^{2}$ & $2.5 \times 10^{2}$ \\
66 & $7.0 \times 10^{1}$ & $1.5 \times 10^{2}$ \\
& & \\
\hline \hline
\end{tabular}

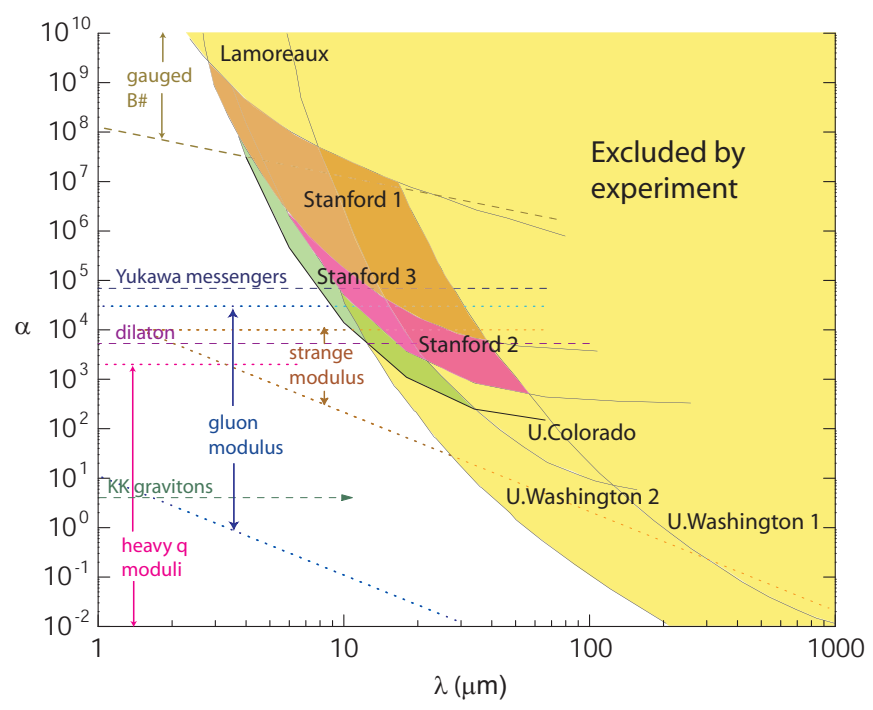

FIG. 9: (color online). New limits on Yukawa-forces. The shaded area refered to as 'Stanford 3' represents the $95 \%$ exclusion region yielded with the present data set, listed in Table $\mathrm{V}$ Experimental limits derived in this work are on $|\alpha|$. The previous results labeled 'Stanford 1' and 'Stanford 2' are presented in references [6] and [7], respectively. Also shown are theoretical predictions and other experimental results as indicated in Refs. [21, 22, 23, 24, 25, 26].

to allow a larger area test mass. Such an improvement is underway in a second-generation rotary-drive experiment [27], expected to be one to two orders of magnitude more sensitive than the present apparatus. A number of other improvements could be implemented resulting in more marginal gains. A doubly-clamped cantilever beam loaded with a test mass in its center could reduce the systematic error associated with the transverse position of the fiber above the test mass. In this configuration, any transverse displacement of the fiber along the test mass length affects the measured vertical displacement to a lesser degree, as the cantilever mode shape is quadratic rather than linear at the mass-attachment point. An additional piezo-electric transducer that, unlike the bimorph, can be operated at the cantilever resonance frequency could be installed near the bimorph to further characterize the effectiveness of the vibration isolation. Voltage noise on the interferometer could potentially be reduced by improving the vibration isolation between the optical fiber in the cryostat and the bimorph actuator. A new set of shield wafers could be fabricated from a double-polished silicon wafer to allow a sharper reflection of the drive mass image during the room temperature alignment procedure. This could potentially reduce uncertainty in the tilt, resulting in decreased effective separation of the masses.

\section{CONCLUSION}

To date, the gravitational interaction remains as one of the least well-understood and least-tested aspects of fundamental physics. In this paper we have presented the latest results from the first-generation Stanford microcantilever experiment, which in sum has improved the limits on new Yukawa-type forces at $20 \mu \mathrm{m}$ by over four orders of magnitude since its conception. Our most recent experimental constraints on Yukawa-type deviations from Newtonian gravity represent the best bound in the range of $5-15 \mu \mathrm{m}$, with a $95 \%$ confidence exclusion of forces with $|\alpha|>14,000$ at $\lambda=10 \mu \mathrm{m}$.

\section{ACKNOWLEDGEMENTS}

We acknowledge many helpful discussions with Dan Rugar and Susan Holmes and thank Savas Dimopoulos for providing much of the motivation for this work. We thank the Bruce Clemens group for assistance with the $\mathrm{Co} / \mathrm{Pt}$ depositions. This work is supported by grant NSF-PHY-0554170.
[1] S. Dimopoulos and G. F. Guidice, Phys. Lett. B 379, 105 (1996).

[2] I. Antoniadis, S. Dimopoulos, and G. Dvali, Nucl. Phys. B 516, 70 (1998).

[3] N. Arkani-Hamed, S. Dimopoulos, and G. Dvali, Phys. Lett. B 429, 263 (1998).
[4] I. Antoniadis, N. Arkani-Hamed, S. Dimopoulos, and G. Dvali, Phys. Lett. B 436, 257 (1998).

[5] N. Arkani-Hamed, S. Dimopoulos, and G. Dvali, Phys. Rev. D 59, 086004 (1999).

[6] J. Chiaverini, S. J. Smullin, A. A. Geraci, D. M. Weld, and A. Kapitulnik, Phys. Rev. Lett. 90, 151101 (2003). 
[7] S. J. Smullin, A. A. Geraci, D. M. Weld, J. Chiaverini, S. Holmes, and A. Kapitulnik, Phys. Rev. D 72, 122001 (2005).

[8] S. Smullin, Ph.D. thesis, Stanford University (2005).

[9] D. Rugar, H. J. Mamin, and P. Guethner, Appl. Phys. Lett. 55, 2588 (1989).

[10] A. Geraci, Ph.D. thesis, Stanford University (2007).

[11] J. Chiaverini, Ph.D. thesis, Stanford University (2002).

[12] Amuneal Manufacturing Corp., 4737 Darrah Street Philadelphia, PA 19124, USA.

[13] G. Bertero, Ph.D. thesis, Stanford University (1996).

[14] Bruce Clemens, private communication.

[15] Quantum Design, Inc., www.qdusa.com Magnetic Property Measurement System.

[16] Epoxi-Patch, Dexter Corporation, 1 Dexter Dr., Seabrook, NH 03874, USA.

[17] A. Lambrecht and S. Reynaud, Eur. Phys. J. D 8, 309 (2000).

[18] R. Kassies, K. O. van der Werf, M. L. Bennink, and C. Otto, Rev. Sci. Instrum. 75, 689 (2004).

[19] T. R. Albrecht, P. Grütter, D. Rugar, and D. P. E. Smith,
Ultramicroscopy 42-44, 1638 (1992).

[20] C. C. Speake and C. T. Trenkel, Phys. Rev. Lett. 90, 160403 (2003).

[21] J. C. Long, H. W. Chan, A. B. Churnside, E. A. Gulbis, M. C. M. Varney, and J. C. Price, Nature 421, 922 (2003).

[22] C. D. Hoyle, D. J. Kapner, B. R. Heckel, E. G. Adelberger, J. H. Gundlach, U. Schmidt, and H. E. Swanson, Phys. Rev. D 70, 042004 (2004).

[23] J. K. Hoskins, R. D. Newman, R. Spero, and J. Schultz, Phys. Rev. D 32, 3084 (1985).

[24] S. K. Lamoreaux, Phys. Rev. Lett. 78, 5 (1997).

[25] S. Dimopoulos and A. A. Geraci, Phys. Rev. D 68, 124021 (2003).

[26] D. J. Kapner, T. S. Cook, E. G. Adelberger, J. H. Gundlach, B. R. Heckel, C. D. Hoyle, and H. E. Swanson, Phys. Rev. Lett. 98, 021101 (2007).

[27] D. M. Weld, J. Xia, B. Cabrera, and A. Kapitulnik, in press. 